\title{
OBESIDAD PEDIÁTRICA: CONCEPTOS BÁSICOS PARA UN ABORDAJE MULTIDISCIPLINARIO EN HONDURAS
}

\author{
Basly Sarhody Rodríguez ${ }^{1}$, Andrea V. Guillén Díaz ${ }^{1}$,Claudia Y. Gradiz \\ Murillo ${ }^{1}$,Wendy D. Martínez Reyes ${ }^{1}$,María Eugenia Sáenz ${ }^{1}$,Cinthia Paola \\ Sagastume Oseguera ${ }^{1}$,Mario F. Rendón Vásquez ${ }^{1}$,Giordana Morales ${ }^{2}$,Candy \\ Hernández ${ }^{2}$ Jazmin Nova ${ }^{2}$ Sheyla Licona ${ }^{1}$ Edda C. Escobar ${ }^{1}$ \\ ${ }^{1}$ Facultad de Ciencias de la Salud, Carrera de Medicina y Cirugía, Universidad Tecnológica Centroamericana, \\ Tegucigalpa Honduras
}

${ }^{2}$ Public and Community Health Department, Liberty University, Virginia, E.U.A

(Enviado: Enero, 2019/Aceptado: Abril, 2019)

\begin{abstract}
Resumen
La obesidad, es la epidemia del siglo XXI, es un problema creciente en la edad pediátrica, los factores que predisponen a esta enfermedad son múltiples, modificables (alta ingesta calórica, bajo gasto energético, psicológicos) y no modificables (genéticos). En el tratamiento, el apoyo de la familia juega un papel muy importante para que este sea exitoso, además, es necesario que se establezcan políticas nacionales de estrategia para la prevención, por lo que es importante conocer las principales causas de obesidad en nuestro país. Este articulo pretende enfocar las diferentes causas que pueden llevar a obesidad en la edad pediátrica, por lo que es necesario realizar un abordaje multidisciplinario para luchar contra esta enfermedad y sus consecuencias.
\end{abstract}

Palabras Claves: Sobrepeso, trastornos nutricionales, índice de masa corporal, terapia combinada

\begin{abstract}
Obesity, is the epidemic of the XXI century, is a growing problem in the pediatric age, the factors that predispose to this disease are multiple, modifiable (high caloric intake, low energy expenditure, psychological) and non-modifiable (genetic). In the treatment, the support of the family plays a very important role for this to be successful, in addition, it is necessary to establish national policies for prevention strategy, so it is important to know the main causes of obesity in our country. This article aims to focus on the different causes that can lead to obesity in the pediatric age, so it is necessary to carry out a multidisciplinary approach to fight against this disease and its consequences.
\end{abstract}

Keywords: Overweight, nutritional disorders, body mass index, combined modality therapy 


\section{Introducción}

La obesidad es la enfermedad del siglo y su prevalencia crece de forma alarmante en niños y adultos, teniendo un gran impacto en la calidad de vida y asociándose con otras patologías como el síndrome metabólico, enfermedades cardíacas, diabetes, dislipidemia y otras. La obesidad es un problema creciente entre la población pediátrica, incluida la hondureña y puede resultar en el desarrollo de múltiples enfermedades a esta edad. La Organización Mundial de la Salud (OMS) define la obesidad como un acumulo de grasa anormal o excesiva de grasa, con valores de peso para la estatura con más de 2 desviaciones estándar y más de 3 desviaciones estándar respectivamente, respecto a las medianas establecidas en los patrones de crecimiento de la OMS (OMS, 1995, 2004). Los indicadores más utilizados para determinar el sobrepeso y la obesidad son el Índice de Masa Corporal (IMC) y el perímetro abdominal.

Sus causas son múltiples e incluyen factores genéticos, psicológicos y sociales. La obesidad en niños también puede estar asociada a factores que son modificables como el sedentarismo, falta de actividades recreativas, el estar por largos periodos frente a la televisión, etc. (Lama More, et al., 2003; OMS, 2016). Además de los factores genéticos, contribuyen el tipo de alimentación que tiene el niño desde el primer día de vida y durante su desarrollo. Si el niño adopta hábitos alimenticios inadecuados, estará más predispuesto a la obesidad y sus consecuencias no sólo físicas, si no que psicológicas como baja autoestima, depresión, etc. A la larga, la obesidad infantil puede disminuir la esperanza de vida o provocar la muerte temprana (Medina, Yuja y Lanza, 2016; OMS, 2010 y 2016).

En los últimos años, el crecimiento de casos de obesidad en niños y adolescentes ha ido en aumento, por lo cual la Asamblea Mundial de la Salud la declaró epidemia del Siglo XXI y aprobó la creación de la Estrategia para la Nutrición, Actividad Física y Prevención de la Obesidad (NAOS), basada en la mejoría del hábito alimenticio, la actividad física diaria y la visión corporal propia (OMS, 2004). La presente publicación enfatiza los aspectos que Honduras necesita enfocar para la lucha integral contra la obesidad infantil y sus consecuencias.

\section{Conceptos Básicos}

Para determinar la categoría de peso en la infancia es importante conocer el IMC (índice de masa corporal), el cual se calcula dividiendo el peso en Kg entre la talla en metros cuadrados $(\mathrm{IMC}=\mathrm{Kg} / \mathrm{m} 2)$. Según la OMS, las curvas y los valores de referencia son necesarios para determinar el IMC: se calculan los valores de Z del IMC para la edad y se clasifica como obesidad la presencia de un valor $Z$ mayor a +3 , sobrepeso entre +2 y +3 y normal de $0 \mathrm{a}-1$ y con déficit por debajo de - 1 (OMS, 1995, 2009 y 2017). Según las Tablas del CDC (Centers for Disease Control and Prevention), el IMC clasifica el estado de peso del niño de acuerdo a su talla y dentro de percentiles como se observa en el Cuadro 1 (Flegal, Ogden, Wei, Kuczmarski y Johnson, 2001). Un percentil de 95 indica sobrepeso (Kuczmarski, et al, 2000; Padula y Salceda, 2008). 
Tabla 1. Índice de Masa Corporal clasificación por percentiles según el Centro para el Control y Prevención de Enfermedades (CDC) de los Estados Unidos.

\begin{tabular}{cl}
\hline Clasificación & Percentil del IMC \\
\hline Bajo peso & Menor de 5 \\
Peso & Entre 5 hasta por debajo \\
saludable & del percentil 85 \\
Sobrepeso & Entre 85 hasta por debajo \\
& de 95 \\
Obeso & Igual o mayor al percentil \\
& 95
\end{tabular}

Fuente: centro para el control y prevención de enfermedades (CDC) Estados Unidos

\section{Epidemiología de la obesidad en niños y jóvenes de Honduras y Latinoamérica}

Según un estudio en escuelas privadas y públicas de la Colonia Kennedy de la Ciudad de Tegucigalpa en el año 2016, se evaluó el peso de 357 niños entre 6 y 11 años de edad de seis escuelas, tres públicas (277 niños) y tres privadas (80 niños). Se encontró que los niños de escuelas privadas tenían mayor frecuencia de sobrepeso y obesidad, con $46.3 \%$ versus $33.2 \%$ de los niños de escuelas públicas.

Tabla 2. Comparación del peso de escolares de varios países latinoamericanos según los percentiles de las curvas de CDC.

\begin{tabular}{|c|c|c|c|c|c|}
\hline $\begin{array}{l}\text { Estado nutricional } \\
\text { según el IMC por } \\
\text { cada muestra }\end{array}$ & $\begin{array}{l}\text { Honduras } \\
\mathrm{N}=357 \\
191 \text { niñas } \\
166 \text { niños }\end{array}$ & $\begin{array}{l}\text { México } \\
\mathrm{N}=418 \\
212 \text { niñas } \\
206 \text { niños }\end{array}$ & $\begin{array}{l}\text { Colombia } \\
\mathrm{N}=326 \\
170 \text { niñas } \\
156 \text { niños }\end{array}$ & $\begin{array}{l}\text { Costa Rica } \\
\text { N=276 } \\
137 \text { niñas } \\
139 \text { niños }\end{array}$ & $\begin{array}{l}106 \text { niñas } \\
104 \text { niños }\end{array}$ \\
\hline Bajo peso & $3 \%$ & - & $1.80 \%$ & $8.60 \%$ & $86.20 \%$ \\
\hline Normal & $54 \%$ & $56.20 \%$ & $60.40 \%$ & $45.30 \%$ & - \\
\hline Sobrepeso & $18 \%$ & $16.20 \%$ & $22.40 \%$ & $28.30 \%$ & $9.50 \%$ \\
\hline Obesidad & $18 \%$ & $27.50 \%$ & $15.30 \%$ & $17.80 \%$ & $4.30 \%$ \\
\hline
\end{tabular}

Fuente: elaboración propia a partir de literatura (Amigo, 2003; Di Gianlippo y Bermúdez, 2013; Evans-Meza y Capitán-Jiménez, 2016; Fajardo Bonilla, 2012; Fuentes et al., 2016; Hernández-Herrera y González-Guajardo, 2014; Ramírez-Izcoa A., et al, 2017).

Una hipótesis planteada fue que los niños de las escuelas privadas podrían tener un mayor poder adquisitivo para comprar comida no saludable en las escuelas (Ramírez-Izcoa, Sánchez-Sierra, Mejía-Irías, Izaguirre González, 2017). Otro estudio hondureño realizado en tres escuelas de las ciudades de Danlí, Comayagua y Salamá, en el cual se estudiaron 609 niños, la mayor parte de los alumnos presentó un estado nutricional normal (66.8\% de 407 niños). Se encontró un importante grupo de niños con sobrepeso (11.8\% de 72 niños), obesidad (15.6\% de 95 niños) y bajo peso 5.7\% (35 niños).4 (Medina Acosta, Yuja y Lanza, 2016). Esto sugiere que el factor social y económico es un predisponente para la obesidad (Sánchez Arena y Ruiz Martínez, 2016). La obesidad en los niños escolares de Latinoamérica se puede ver de forma general en el Cuadro No. 2, el cual muestra niveles preocupantes en México (27.5\% de los escolares de la 
muestra estudiada), seguido por Honduras (18\%). Estos estudios fueron realizados en escuelas públicas y privadas (Amigo, 2003; Di Gianlippo y Bermúdez, 2013; Evans-Meza y CapitánJiménez, 2016; Fajardo Bonilla, 2012; Fuentes et al., 2016; Hernández-Herrera y GonzálezGuajardo, 2014; Ramírez-Izcoa A., et al, 2017).

\section{Factores que predisponen a obesidad en niños}

Los factores que predisponen a la obesidad infantil son múltiples, desde factores no modificables (genéticos y endocrinos), hasta factores modificables que tienen que ver con el estilo de vida; el sedentarismo y la alimentación (Figura 1). Todos estos factores deben ser abordados adecuadamente (CDC, 1997).

\section{Factores nutricionales}

La buena nutrición debe iniciar desde la vida intrauterina con la buena alimentación de la madre durante la gestación (Martínez, Navarrete, Rodríguez y Herrera, 2012). También es clave la adquisición de buenas prácticas alimenticias del lactante desde el inicio de la alimentación complementaria y en etapas posteriores. En edades siguientes, los malos hábitos pueden predisponer a un rebote de adiposidad en una edad más temprana, elevando el riesgo de sobrepeso y obesidad en la infancia tardía o en la edad adulta (Chueca, Azcona y Oyarzábal, 2002; Martínez, Navarrete, Rodríguez y Herrera, 2012).

\section{Sedentarismo}

El sedentarismo se ha relacionado últimamente con el uso constante de aparatos electrónicos como el celular, computadoras, tableta, televisión y el reducido espacio recreativo son factores que limitan una actividad física adecuada para el niño. La dieta o patrón alimenticio que el niño tiene, depende de lo que es ofrecido por los padres, quienes muchas veces les proporcionan alimentos no ideales, como las comidas rápidas contenientes de altos nivel calóricos y pocos o ningún tipo de nutrientes. También es inapropiado el hábito de darles bebidas gaseosas que son fuentes de altos contenidos de azúcar (Cardona, 2016). Todos estos factores desarrollan en el niño y el adolescente conductas que serán difícil de modificar si no se toman las medidas adecuadas para cambiarlas (OMS, 1995; Saavedra y Dattilo, 2012).

\section{Factores genéticos}

Se estima que aproximadamente $25 \%$ a $40 \%$ del índice de masa corporal tiene carácter hereditario, requiriendo de alteraciones de esos componentes externos para expresar un efecto (Sahoo et al, 2015). La susceptibilidad al sobrepeso podría ser un rasgo hereditario, acentuada durante los momentos de cambios en el balance energético asociados con aspectos del medio ambiente, socioeconómicos y culturales que alteran la ingesta y expensa de alimentos. La asociación de la obesidad con síndromes genéticos específicos es un campo de investigación amplio, incluso ligando alteraciones endocrinológicas y neurológicas. Ejemplos de estos son los síndromes como Prader Willi o el de Klinefelter, que representan menos del cinco por ciento de los casos de obesidad por causa endógena y que ya son diagnosticados en nuestra región.

El síndrome de Prader Willi se caracteriza por una disfunción del hipotálamo responsable de diversos cambios metabólicos originados por la deleción de genes en el cromosoma 15q11.2q13. (Angulo, 2015; Butler, 2016). Otro síndrome llamado Bardet- Biedl se caracteriza por retraso verbal, alteraciones digitálicas y un rápido incremento en el peso, que es más notable en 
los lactantes mayores (Martos, Serra, Pérez y Argente, 2017; Geets, Meuwissen y Van Hul, 2019). Estos pacientes presentan múltiples mutaciones en varios genes responsables del efecto neuronal sobre la ingesta de comida y la saciedad.

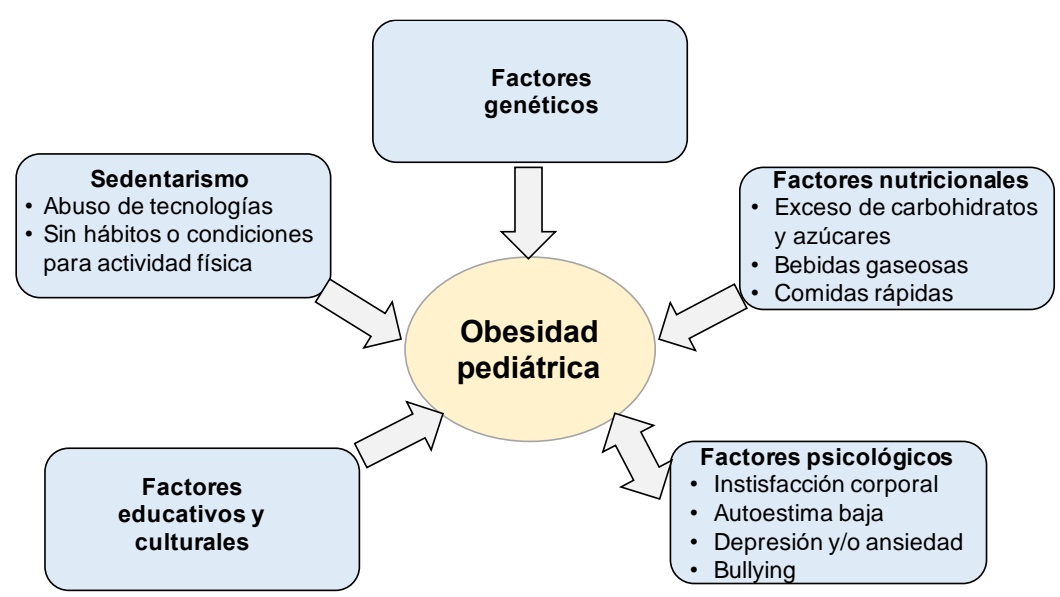

Figura 1: Factores que predisponen a obesidad pediátrica.

Fuente: elaboración propia según conceptos mencionados en factores que predisponen a obesidad en niños

El síndrome de Alstrom presenta mutaciones en el gen codificador para la Proteína 1 del Síndrome de Alstrom (ALMS1) en el cromosoma 2p13. Las manifestaciones son similares a las del Bardet- Biedl pero sin presencia de retraso. El síndrome de Carpenter presenta alteraciones en el gen RAB23 del cromosoma 6p11 y cursa con obesidad, alteraciones digitálicas y craneosinostosis (Martos, et al., 2017; Geets et al., 2019; Lu y Loos, 2013).

Las deficiencias en la producción y circulación de la leptina también son responsables de obesidad extrema congénita. Se ha detectado múltiples alteraciones en el gen humano de la leptina (LEP) localizado en el cromosoma 7q31.3 (Funcke et al, 2014).

Tabla 3. Genes de la leptina relacionados con la obesidad.

\begin{tabular}{|c|c|c|c|}
\hline & & & \\
\hline POMC & Pro-opiomelanocortina & KLF9 & $\begin{array}{l}\text { Factor } 9 \text { Kruppel-like, } \\
\text { relacionado con el IMC }\end{array}$ \\
\hline MC4R & $\begin{array}{l}\text { Receptores de la } \\
\text { melanocortina (MC), la } \\
\text { forma } 4 \text { (MC4R) es la más } \\
\text { comúnmente afectada }\end{array}$ & TOMM40 & $\begin{array}{l}\text { Translocasa de la membrana } \\
\text { mitocondrial externa } \\
\text { homólogo } 40\end{array}$ \\
\hline $\operatorname{PPAR} \gamma$ & $\begin{array}{l}\text { Factor de transcripción } \\
\text { receptor proliferador de } \\
\text { peroxisomas activados } \\
\text { gamma }\end{array}$ & LYPLAL1 & $\begin{array}{l}\text { Liso-phospholipase-like } 1 \text {, } \\
\text { relacionado con tejido } \\
\text { adiposo subcutáneo }\end{array}$ \\
\hline Locus FTO & $\begin{array}{l}\text { fat mass and obesity } \\
\text { associated gene, es el más } \\
\text { relacionado con la obesidad }\end{array}$ & NEAR Spry2 & $\begin{array}{l}\text { Relacionado con el } \\
\text { porcentaje de grasa corporal }\end{array}$ \\
\hline
\end{tabular}

Fuente: elaboracion propia según conceptos de Martos et al (2017), Geets et al (2019), Funcke et al (2014). 
Se han estudiado deleciones, transverciones y transiciones tanto de aminoácidos en particular así como de uno o más codones responsables del daño en la LEP (Cuadro 2) (Lu y Loos, 2013).

\section{Efectos de la obesidad en los niños}

\section{Psicológicos}

Si el niño crece en un ambiente donde no se le enseña estilos de vida saludable e imágenes corporales saludables, el niño será propenso a desarrollar obesidad. Esto resulta en burlas, rechazo, baja autoestima, ansiedad y depresión. Estos trastornos podrían marcar psicológicamente al niño por el resto de su vida (González-Toche, et al., 2017). El niño aprende la autorregulación a medida que crece, cuando esa autorregulación es interrumpida por alguno de los trastornos psicológicos antes mencionados, el niño comenzará a perder esas capacidades de autorregulación y perderá la capacidad de reconocer sensaciones de hambre y saciedad (Hernández-Arteaga, Rosero-Galindo y Montenegro Coral, 2015).

Como resultado de ese mismo ambiente no saludable, los niños son propensos a manifestar alteraciones en el aprendizaje, inseguridades, resistencia a actividades recreativas, problemas de comportamiento y otros aspectos psicoconductuales (González-Toche, et al., 2017). A la larga, todos esos sucesos generarán una personalidad vulnerable y hábitos en el niños que le convertirán en un adulto con diversas comorbilidades como hipertensión, diabetes, síndrome metabólico y otros.

\section{Bullying}

El padecer de obesidad vuelve al niño más propenso a sufrir de burlas. Los escolares que tienen sobrepeso son menos capaces de realizar actividades físicas y sociales. Estas situaciones impiden su desarrollo adecuado (González-Toche, et al., 2017; Rodríguez y González, 2013). Un estudio realizado en la Provincia de Granada evaluó la victimización e imagen corporal en escolares. Se demostró que aquellos escolares que se percibían con obesidad o sobrepeso eran mucho más victimizados y más propensos a sufrir acoso verbal y físico (Chacón Cuberos, Gonzáles Valero, Padial Ruz y Castro Sánchez, 2017).

\section{Baja autoestima}

Una de las consecuencias a corto plazo en la obesidad infantil es la disminución del autoestima. Del mismo modo, la pérdida de la autoestima puede llevar a un niño a la obesidad. Los niños con obesidad están más expuestos a sufrir discriminación social por burlas, también sufren de marginación por su aspecto físico y por su capacidad física disminuida. Esto va de la mano con otros aspectos psicológico como la depresión (Fajardo Bonilla, 2012; Acosta-Hernández, et al, 2013).

Es necesario crear las condiciones para que los niños con sobrepeso tengan un equilibrio entre salud física y mental para que desarrollen un mejor autoconcepto, disminuyendo así la depresión, la ansiedad o los intentos suicidas. Los niños obesos tienen miedo a ser personas poco valoradas y rechazadas, lo cual lleva los lleva a sentirse tristes, aislados y nerviosos. Todo lo anterior también provoca una ingesta de alimentos excesiva, ya que comer sirve de refugio para disminuir el nivel de ansiedad y como auto compensación por sentirse inferior o incapaz. Esta situación se ha convertido en un grave problema, especialmente en el ámbito escolar. 
Muchos adultos creen equivocadamente, que al realizar comentarios negativos y al desvalorarlos, motivaran al niño a que baje de peso. Sin embargo, eso más bien tendrá un efecto contrario, agravará el problema favoreciendo el deterioro de la autoestima y la imagen corporal. Hoy en día hay un gran énfasis tergiversado sobre el aspecto físico, condicionando a que en un futuro los niños presenten trastornos alimentarios como anorexia, bulimia, dismorfia corporal, obesidad (Padula y Salceda, 2008).

\section{Insatisfacción corporal}

Las valoraciones sobre la imagen corporal propia inician desde la etapa escolar (6-12 años de edad), pero puede ocurrir antes en algunos casos (Jiménez Flores, Jiménez Cruz y Bacardí Gascón, 2017). La insatisfacción corporal puede conducir a prácticas no nutricionales que aparentan orientar a una mejoría en cuanto al peso e imagen, por ejemplo, hacer ayunas, evitar tiempos de comida, inducir vómitos y el uso de laxantes (Monteiro, Novaes, Santos y Fernándes, 2014). Los cambios en la regulación adiposa que trae consigo la pubertad de las niñas, podría determinar porque se manifiesta más temprano en ellas la inseguridad e insatisfacción. La presión social sobre cómo deben de ser los cuerpos ideales de hombres y mujeres también tiene un valor sobre la insatisfacción corporal, así como en un daño al autoestima y riesgo a depresión (Sánchez y Ruiz, 2015).

\section{Depresión}

La ansiedad y la depresión tienen una amplia gama de consecuencias para el adolescente y pueden servir como precursores para el desarrollo de trastornos de la alimentación. La forma de presentación de la depresión es variada, pero destacan conductas y síntomas característicos entre ellos; humor disfórico, agresividad, desesperación, trastornos del sueño, disminución en el rendimiento escolar y poca sociabilidad. (López Morales, Pasacalis, Gonzáles Heredia y Brito Zurita, 2014). Es importante hacer un abordaje multidisciplinario incluyendo un abordaje desde el punto de vista psicológico para ayudar a los niños a modificar sus malos hábitos y mejorar su calidad de vida.

\section{Tratamiento integral en la obesidad infantil}

En el abordaje multidisciplinario, lo esencial es combatir la causa de la obesidad, sea por aumento de la ingesta calórica, problemas genéticos, factores psicológicos o por el bajo gasto calórico. Es importante tener el apoyo de toda la familia para que el manejo sea exitoso. Se debe modificar los hábitos en todo el entorno del niño tanto como sea posible (Burrows, 2000). La recomendación de la Academia Norteamericana de Pediatría es cambiar los hábitos de la ingesta y mantener la actividad física. La disminución de peso en la población pediátrica tiene la ventaja de que a medida va creciendo el niño, la cantidad de peso a perder es menor. Una pérdida de peso de 10 a 20 gramos al día hace que los resultados sean efectivos. En los niños la pérdida de peso es más difícil que en adultos, ya que a menor edad no se tiene una perspectiva del problema (Norero y Rosselot, 2001). 


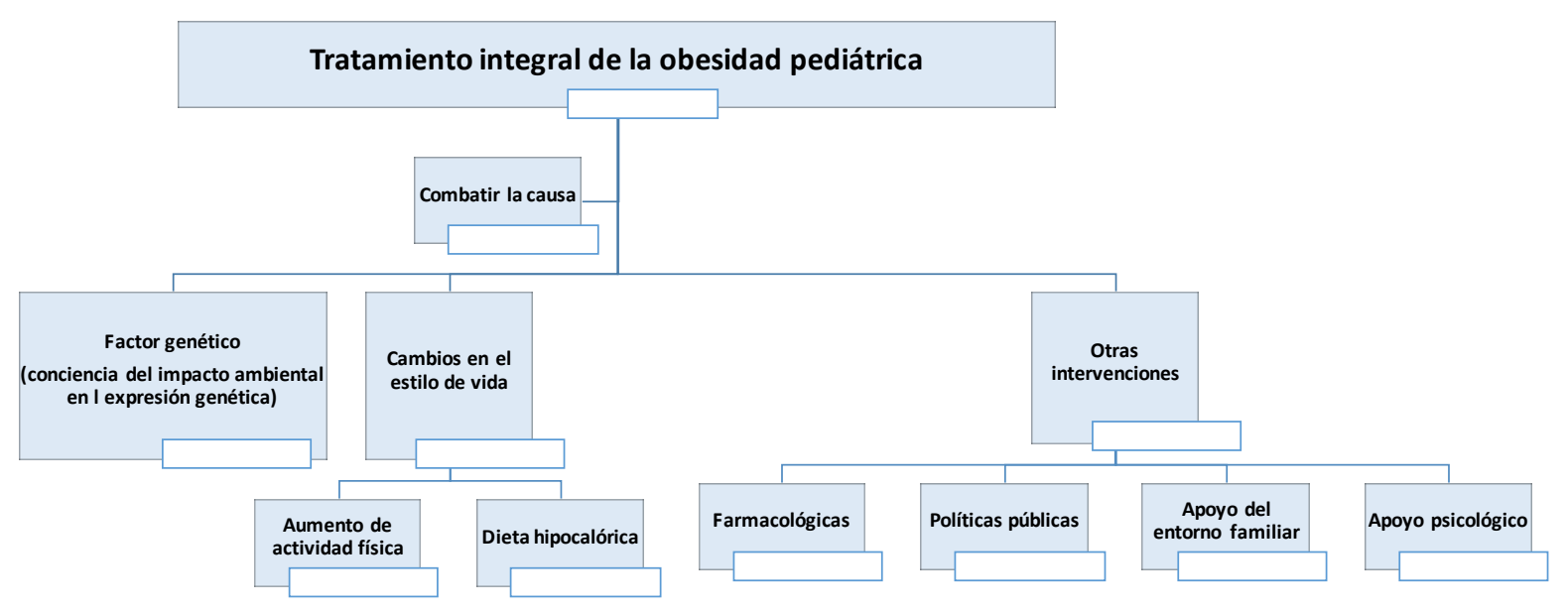

Figura 2: Intervenciones para el tratamiento integral de la obesidad infantil Fuente: elaboración propia según conceptos de Burrows (2000), Norero y Rosselot (2001).

Actualmente, los niños y adolescentes tienen acceso a muchas tecnologías, aunque esto puede ser un obstáculo para que hagan actividad física, también puede ser un instrumento de concientización. Entonces, el tratamiento exitoso incluye mantener una dieta hipocalórica, ir aumentando la actividad física periódicamente hasta llegar a la meta deseada y favorecer la participación del entorno familiar para una mejor salud de los niños (Burrows, 2000; Norero y Rosselot, 2001).

\section{Estrategias para reducir la obesidad pediátrica en Honduras}

\section{Políticas para una niñez saludable}

La Secretaria de Salud en Honduras cuenta con varios programas que incluyen la promoción de la salud para la población infantil, como el "Programa Presidencial de Salud, Educación y Nutrición" y el Programa "Escuelas Saludables". ${ }^{34}$ (Secretaría de Salud en Honduras, 2014). Estos programas están enfocados predominantemente a la prevención de la desnutrición, la cual ha sido por mucho tiempo causa de muerte en Honduras. Sin embargo, estas políticas no habían enfocado hasta ahora el problema de la obesidad pediátrica ya que ésta fue considerada por mucho tiempo como un problema del adulto. ${ }^{35}$ (Ariza C, et al. 2015). Es necesaria la revisión de dichas políticas y respectivos presupuestos para Honduras.

\section{Lactancia materna}

La lactancia materna exclusiva hasta los 6 meses es un mecanismo de prevención importante para la obesidad pediátrica y otras enfermedades como asma, neumonías y otras infecciosas. Según la OMS, después de los 6 meses de debe de agregar alimentos suplementarios como otros tipos de leche y alimentos sólidos. Sin embargo, muchas de las formulas artificiales que se encuentran en el mercado tienen altos niveles proteicos que son inadecuados para los recién nacidos y lactantes menores, pudiendo aumentar el riesgo de obesidad en el futuro (Aguilar Cordero et al., 2015; OMS, 2017). Según la OMS, existe un riesgo menor de obesidad en niños y adolescentes que fueron amamantados; existiendo un efecto dosis-respuesta: a mayor duración de lactancia materna menor riesgo de obesidad (OMS, 2010).

\section{Actividad física}

Es una necesidad que los padres o encargados con autoridad disminuyan las horas que los niños pasan frente al televisor, computadora u otra tecnología que disminuya el gasto calórico por 
actividad física (Trejo Ortiz, Chairez, Mollinedo Montaño, Lugo Balderas, 2012). Se deben implementar estrategias a nivel escolar, como aumentar las horas de clase de educación física apropiada a la edad y que realicen actividades que impliquen un mayor gasto calórico. Es esencial que en una edad temprana mostremos al niño como disfrutar de la actividad física durante su tiempo libre para que se convierta en un estilo de vida y no una obligación. Es aconsejable realizar ejercicios 30 a 60 minutos al día mínimo tres veces por semana para mantener la masa muscular mientras se somete a un programa de restricción calórica, tomando en cuenta que la actividad física se debe aumentar gradualmente para que el organismo se adapte adecuadamente a la intensidad (García-Hermoso, Escalante, Domínguez y Saavedra, 2013).

\section{Alimentación saludable y accesible}

El perfil alimentario de los niños con obesidad incluye generalmente el consumo elevado de productos de repostería, pastelería y refrescos azucarados y un aporte disminuido de frutas y verduras. Es importante educar en buenas prácticas nutricionales en la casa y la escuela, así como brindar acceso a los nutrientes necesarios para cubrir con las demandas metabólicas del organismo del niño (Figura No. 3) (Cardona Gómez, 2016) Debe realizarse un régimen de comidas variadas, apetecibles y adaptadas al niño y su familia. La dieta no tiene que ser prefabricada, la misma se debe generar en base a la edad, sexo y actividad. Por otro lado, es deber del Estado favorecer la seguridad alimentaria de la población y dar prioridad a la población escolar.

\section{Educación nutricional a padres y cuidadores}

La educación a los padres en particular, puede ser difícil, ya que algunos estudios indican que hay madres que siguen creyendo que los niños "gorditos" son muestra de salud (Hochdorn, et al., 2018). A los padres y cuidadores hay que darles a conocer qué tipo de alimentos deben ingerir sus hijos, con qué frecuencia deben comer, cuál es el beneficio que aporta cada alimento y con qué regularidad se debe consumir. Aunque los padres tienen una gran influencia dietética, los niños pueden desarrollar un control inmediato de sus propios comportamientos, como participar en actividades físicas, elegir hábitos más sedentarios o elegir cuáles alimentos comerán si hay opciones disponibles.

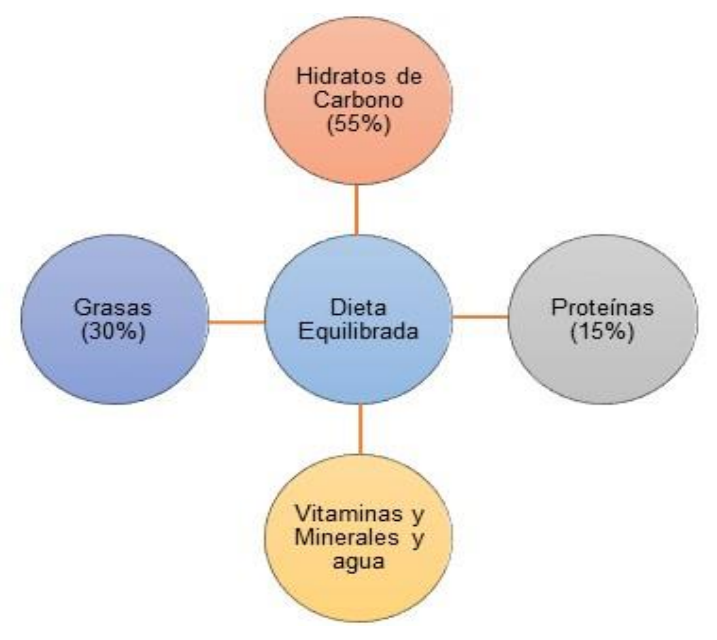

Figura 3: Elementos para una dieta equilibrada.

Fuente: elaboración propia según conceptos de Cardona (2016). 
Por esta razón, es importante educar a los niños sobre buenos hábitos de alimentación y de vida desde una edad temprana, con la esperanza de que la información presentada ayude a guiar las decisiones a través de su desarrollo hasta la edad adulta y permita una buena elección de vida a largo plazo. Esta educación en salud y nutrición debería ser parte la educación primaria (Hills, Dengel y Lubans, 2015).

\section{Conclusiones}

Desde una perspectiva de salud pública, se requiere educación a padres, niños, maestros y que el Estado brinde seguridad alimentaria a la población pediátrica y que establezca políticas públicas para la prevención y control de la obesidad. Para generar un cambio de comportamiento preventivo de la obesidad, es muy importante que cada miembro de la familia debe participar activamente en lugar de centrarse únicamente en los niños o en los padres. Las escuelas son un excelente punto de apoyo al trabajo de los padres. En la estrategia integral contra la obesidad pediátrica, debe haber un abordaje multidisciplinario regido por políticas públicas y sanitarias actualizadas e implementadas con efectividad.

Es importante reconocer los principales causales de obesidad y sobrepeso entre la población pediátrica en nuestro país para que el abordaje sea el adecuado y los resultados al final sea los esperados además es claro y de suma importancia el estudio del estilo de vida en cuanto a salud ya que este permitirá reconocer factores como hábitos alimenticios, actividad física que será vitales para mejorar la calidad de vida del paciente.

\section{Agradecimiento}

A la Dra. Reyna M. Durón, Docente Investigadora de la Facultad de Ciencias de la Salud, por su asesoría y ediciones a este manuscrito.

\section{Conflicto de intereses}

Los autores no tienen ningún conflicto de interés relacionado a este trabajo.

\section{Contribuciones}

Todos los autores participaron en la revisión biliográfica y redacción del texto, GM, CH y ECE hicieron la edición final del manuscrito.

\section{Referencias bibliográficas}

Acosta-Hernández, M., Gasca-Pérez, E., Ramos-Morales, F. R., et al. (2013). Factores, causas y perspectivas de la obesidad infantil en México. Revista de los Estudiantes de Medicina de la Universidad Industrial de Santander, 26, 59-68.

Aguilar Cordero, M. J., Sánchez López, A. M., Madrid Baños, N., Mur Villae, N., Expósito Ruiz, M., Hermoso Rodríguez, E. (2015). Lactancia materna como prevención del 
sobrepeso y la obesidad en el niño y el adolescente; revisión sistemática. Nutr Hosp, 31, 606-620. http://dx.doi.org/10.3305/nh.2015.31.2.8458

Amigo, H. (2003). Obesidad en el niño en América Latina: situación, criterios de diagnóstico y desafíos. Cad Saúde Pública, 19, S163-S170.

Fuentes, C. A., Guerra, J. J., Abujieres, C. M., Coca, C. J., Alvarenga, D. F., Rodríguez, J. E., \& Murcia, M. R. (2016). Prevalencia de obesidad en escolares en dos comunidades rurales. Revista Científica de la Escuela Universitaria de las Ciencias de la Salud, 3(1), 25-29.

Angulo, M. A., Butler, M. G., Cataletto, M. E. (2015). Prader-Willi syndrome: a review of clinical, genetic, and endocrine findings. J Endocrinol Invest, 38, 1249-1263. $10.1007 / \mathrm{s} 40618-015-0312-9$

Ariza, C., Ortega Rodríguez, E., Sánchez Martínez, F., Valmayor, S., Juárez, O., Pasarín, M. I. (2015). La prevención de la obesidad infantil desde una perspectiva comunitaria. Aten Primaria, 47, 246-255.

Butler, M. G., Lee, J., Cox, D. M., Manzardo, A. M., Gold, J. A., Miller, J. L.,.. Driscoll DJ. (2016). Growth charts for Prader-Willi Syndrome during growth hormone treatment. Clin Pediatr (Phila), 55(10), 957-974. doi: 10.1177/0009922815617973.

Burrows, A. R. (2000). Prevención y tratamiento de la obesidad desde la niñez: la estrategia para disminuir las enfermedades crónicas no transmisibles del adulto. Rev Méd Chile, 128, 105-110. http://dx.doi.org/10.4067/S0034-98872000000100015

Cardona Gómez, J. (2016). Actividad física y factores de riesgo cardiovascular en niños y adolescentes. Revista de Educación Física, 5, 70-85.

CDC (Centers for Disease Control and Prevention). (1997). Guidelines for school and community programs to promote lifelong physical activity among young people. MMWR Recomm Rep, 46(RR-6), 1-36. 
Chacón Cuberos, R., Gonzáles Valero, G., Padial Ruz, L., Castro Sánchez, M. (2017). Relación entre victimización escolar e imagen corporal en escolares de la provincia de Granada. Eur J Develop Educa Psychop, 5, 35-44.

Chueca, M., Azcona, C., Oyarzábal, M. (2002). Obesidad infantil. Anales Sis San Navarra, 25, (Supl. 1), 127-141.

Rodríguez, C., Gonzáles, A. (2013). Abordaje familiar y psicológico del niño con obesidad exógena. Academia Mexicana de Cirugía, 241-250.

Di Gianlippo, M., Bermúdez. (2013). Prevalencia de sobrepeso y obesidad en escolares de la Parroquia Coquivacoa del Municipio Maracaibo - Venezuela. Revista Latinoamericana de Hipertensión, 8, 68-76.

Evans-Meza, R., Capitán-Jiménez, C. (2016). Prevalencia de sobrepeso y obesidad en niños de escuelas de Santo Domingo de Heredia, Costa Rica. Rev Hisp Cienc Salud, 2, 12-20.

Fajardo Bonilla, A. (2012). La prevalencia de sobrepeso y obesidad, consumo de alimentos y patrón de actividad física en una población de niños escolares de la ciudad de Bogotá. Rev Fac Med, 20, 112-116.

Flegal, K. M., Ogden, C. L., Wei, R., Kuczmarski, R. L., Johnson, C. L. (2001). Prevalence of overweight in US children: Comparison of US growth charts from the Centers for Disease Control and Prevention with other reference values for body mass index. Am J Clin Nutr, 73(6),1086-1093. https://doi.org/10.1093/ajcn/73.6.1086

Funcke, J.B., von Schnurbein, J., Lennerz, B., et al. (2014). Monogenic forms of childhood obesity due to mutations in the leptin gene. Molecular and Cellular Pediatrics, 1, 3. doi: 10.1186/s40348-014-0003-1.

García-Hermoso, A., Escalante, Y., Domínguez, A. M., Saavedra, J. M. (2013). Efectos de un programa de ejercicio y dieta en niños obesos: un estudio longitudinal. Journal of Sport and Health Research, 5, 273-282. 
González-Toche, J., Gómez-García, A., Gómez-Alonso, C., Álvarez-Paredes, M. A., ÁlvarezAguilar C. (2017). Asociación entre obesidad y depresión infantil en población escolar de una unidad de medicina familiar en Morelia, Michoacán. Aten Fam, 24, 8-12.

Hernández-Arteaga, I., Rosero-Galindo, C. Y., Montenegro Coral, F. A. (2015). Obesidad: una pandemia que afecta a la población infantil del siglo XII. Curare, 2, 37-38.

Hernández-Herrera, J., González-Guajardo, E. (2014). Prevalencia de sobrepeso y obesidad en niños de Monterrey, Nuevo León. Rev Med Inst Mex Seguro Soc, 52, 42-47.

Hills, A., Dengel, D., Lubans, D. (2015). Apoyo a las prioridades de salud pública: recomendaciones para la educación física y la promoción de la actividad física en las escuelas. Progreso en Enfermedades Cardiovasculares, 57, 368-374.

Hochdorn, A., Faleiros, V. P., Camargo, B. V., Bousfield, A. B., Wachelke, J. F., Quintão, I. P.,... Gregori, D. (2018). Obese children are thin in parents' eyes: A psychologically, socially, or culturally driven bias? J Health Psycho, 23(1), 114-126. doi: $10.1177 / 1359105316676328$.

Jiménez Flores, P., Jiménez Cruz, A., Bacardí Gascón, M (2017). Insatisfacción con la imagen corporal en niños y adolescentes: revisión sistemática. Insatisfacción corporal, autoestima y depresión en niñas con obesidad. Nutr Hosp, 34, 479-489.

Kuczmarski, R. J., Ogden, C. L., Grummer-Strawn, L. M., Flegal, K. M., Guo, S. S., Wei, R.,... Johnson, C. L. (2000). CDC growth charts: United States. Adv Data, (314), 1-27.

Lama More RA, Franch AA, Gil-Campo M, et al. Obesidad Infantil. Recomendaciones del Comité de Nutrición de la Asociación Española de Pediatría Parte I. Prevención. Detección precoz. Papel del Pediatra. An Pediatr (Barc) 2006;65(6):607-15.

Geets, E., Meuwissen, M. E. C., Van Hul, W. (2019). Clinical, molecular genetics and therapeutic aspects of syndromic obesity. Clin Genet, 95(1), 23-40. doi: 10.1111/cge.13367.

López Morales, M., Pasacalis Orozco, J., Gonzáles Heredia, R., Brito Zurita, O. Depresión y estado de nutrición en escolares de Sonora. Rev Med Inst Mex Seguro Soc 2014;52(Supl 1):S64-S67. 
Lu, Y., Loos, R. J. F. (2013). Obesity genomics: assessing the transferability of susceptibility loci across diverse populations. Genome Medicine, 5(6), 55. doi: 10.1186/gm459

Martínez AP, Navarrete GE, Rodríguez LC, Herrera RR. (2012). Alteraciones en la nutrición fetal y en las etapas tempranas de la vida. Su repercusión sobre la salud en edades posteriores. Acta Pediatr Mex, 33(1), 26-31. doi: 10.18233/APM33No1pp26-31

Martos Moreno, G., Serra Juhe, C., Pérez Jurado, L., Argente, J. (2017). Aspectos genéticos de la obesidad. Rev Esp Endocrinol Pediatr, 8(Suppl), 21-32.

Medina Acosta, C., Yuja, N., Lanza Martínez, O. (2016). Prevalencia de sobrepeso y obesidad en niños de 6 a 12 años de tres escuelas de Honduras. Archivos de Medicina, 12(13).

Monteiro, L. A., Novaes, J. S., Santos, M. L., Fernándes, H. M. (2014). Body dissatisfaction and self-esteem in female studentsaged 9-15: The effects of age, family in come, body mass index levels and dance practice. Journal of Human Kinetics, 43, 25-32. doi 10.2478/hukin-2014-0086

Norero, V. C, Rosselot, J. E. (2001). Investigación clínica en Chile: ¿Cómo superar una inquietante evolución? Rev Méd Chile, 129(3), 317-323.

Organización Mundial de la Salud. (1995). El estado físico: uso e interpretación de la antropometría. Informe de un Comité de Expertos. Ginebra.

Organización Mundial de la Salud. Estrategia mundial sobre régimen alimentario, actividad física y salud. Ginebra, 2004, A57/9.

Organización Mundial de la Salud. Informe de la Comisión para acabar con la obesidad infantil. Ginebra, 2016.

Organización Mundial de la Salud. La alimentación del lactante y del niño pequeño. Ginebra, 2010, p.120.

Organización Mundial de la Salud. (2009). Patrones de Crecimiento del Niño de la OMS.

Curso de Capacitación sobre la Evaluación del Crecimiento del Niño. Ginebra.

Recuperado de https://www.who.int/childgrowth/training/es/ 
Organización Mundial de Salud. Metas mundiales de nutrición 2025. Documento normativo sobre lactancia materna. Ginebra 2017, p.8.

Padula, G., Salceda, S. A. (2008) Comparison between references of the overweight and obesity prevalence, through the Body Mass Index, in Argentinean children. ALAN, 58(4), 330-335.

Ramírez-Izcoa, A., Sánchez-Sierra, L., Mejía-Irías, C., Izaguirre González, A. (2017). Prevalencia y factores asociados a sobrepeso y obesidad infantil en escuelas públicas y privadas de Tegucigalpa, Honduras. Rev Chil Nutr, 44(2),

Saavedra, J. M., Dattilo, A. M. (2012). Factores alimentarios y dietéticos asociados a la obesidad infantil: recomendaciones para su prevención antes de los dos años de vida. Rev Perú Med Exp Salud Pública, 29(3), 379-85.

Sahoo, K., Sahoo, B., Kumar Choudhury, A., Nighat Yasin, S., Kumar, R., Singh Bhadoria, A. (2015). Childhood obesity: causes and consequences. J Family Med Prim Care, 4(2), 187-192. doi: 10.4103/2249-4863.154628

Sánchez Arenas, J. J., Ruiz Martínez, A. O. (2015). Relación entre autoestima e imagen corporal en niños con obesidad. Rev Mex de Trastor Aliment, 6(1), 38-44.

Secretaría Salud de Honduras. Plan Nacional de Salud 2014-2018. 2014 p.45.

Trejo Ortiz P, Chairez S, Mollinedo Montaño F, Lugo Balderas L. Relación entre actividad física y obesidad en escolares. Revista Cubana de Medicina General Integral. 2012;28(1):34-41. 